\title{
Psychische Störungen bei Straftätern
}

\section{Forensisch relevante Krankheitsbilder}

Günter Hinrichs und Denis Köhler

"K rank und/ kriminell « lautete das Thema der 18. (forensischen) Eickelborner Fachtagung im Jahre 2003 und ging damit auf ein Gutachten von Rasch aus dem Jahre 1983 zurück, welches die Grundlage für die Entwicklung einer eigenständigen forensischpsychiatrischen Klinik in Lippstadt-Eickelborn bildete. Drei Jahre später äußerte sich Rasch (1986) eingehend $\mathrm{zu}$ forensisch relevanten psychologisch-psychiatrischen Störungsbildern und formulierte seinen strukturell-sozialen Krankheitsbegriff. Danach soll für einen Straftäter beurteilt werden, ob ein bestimmter Zustand bei ihm die Struktur von »Krankheit « habe und ob er die allgemeine soziale Kompetenz (Dimensionen: Einengung der Lebensführung, Arbeitsunfähigkeit, Abbruch bzw. Verlust von Kontakten, verzerrte Realitätsbeurteilung, Stereotypisierung des Verhaltens, festgelegt sein auf bestimmte Verhaltensmuster, Häufung sozialer Konflikte auch außerhalb strafrechtlicher Belange) der Persönlichkeit beeinträchtige (Rasch 1986, S. 43, 44). Einfach gesprochen werden damit psychische und soziale Komponenten für Kriminalität in einem Stufenmodell verbunden, wobei psychologisch-psychiatrische Handicaps quasi die Schädigungsgrundlage bilden, aus der sich ein Sozialversagen mit Affinität zu strafbarem Verhalten ergibt. Auch wenn dieser Krankheitsbegriff unter wissenschaftlichen Kriterien deutliche Schwächen aufweist, gelingt ihm in zusammenfassender Betrachtung die Berücksichtigung psychischer und sozialer Phänomene in Bezug auf kriminelles Verhalten.

\section{Historische Entwicklung}

Betrachtet man die geschichtliche Entwicklung, so ist die Berücksichtigung psychischer Phänomene in Verbindung mit Kriminalität relativ neu. Zwar wurden gerade bei ausgefallenen Straftaten immer wieder seelische Begleitumstände in Einzelfällen herausgestellt, eine systematische Betrachtung setzt jedoch erst zu Beginn des letzten Jahrhunderts mit der Psychoanalyse ein. Freud (1916) beschrieb den sog. neurotischen Verbrecher aus Schuldbewusstsein noch unter recht mechanistischen Vorstellungen, schon mit Aichhorns (1926) Arbeiten ist eine Erweiterung um pädagogische Aspekte verbunden. Unter dem Be- mühen um eine Differenzierung des Verwahrlosungs- bzw. Dissozialitätsbegriffes entstand die Vorstellung der neurotischen Delinquenz prototypisch etwa als wiederholter Kaufhausdiebstahl von Dingen, die weder dem eigenen Nutzen noch dem materiellen Gewinn dienen, rasch entdeckt werden, ggf. unter demonstrativem Verhalten, die seelische Erschütterung scheint dann oberflächlich zu kontrastieren mit dem Wiederholungszwang. Aber auch schwerwiegende Delikte wie aggressive Beziehungs- und Konflikttaten sowie einige Sexualstraftaten werden mit diesem Begriff gekennzeichnet, der sozusagen die spezifische Ätiologie einer strafbaren Handlung unter besonderer Berücksichtigung psychischer Konflikte aufzeigt. Neuere psychoanalytische Vorstellungen beschäftigen sich unter psychodynamischen Gesichtspunkten auch mit der chronisch-dissozialen Entwicklung, womit eine Verbindung zu den Persönlichkeitsstörungen hergestellt wird.

\section{Der medizinisch-psychiatrische Krankheits- begriff der forensischen Psychiatrie}

Die Beschäftigung der forensischen Psychiatrie mit Straftätern und kriminellem Verhalten bestand lange Zeit in entsprechender gutachterlicher Tätigkeit unter besonderer Fragestellung und der Verwahrung der psychiatrisch erkrankten Rechtsbrecher mit Gefährdungspotential, wobei unter pädagogischen Gesichtspunkten Intelligenzgeminderte mit einbezogen wurden. Als handlungsleitend galt ein medizinisch-psychiatrischer Krankheitsbegriff, wie er sich auch in den Überlegungen von Rasch (1986) noch findet. Eine Veränderung setzte mit der Verbreitung der bekannten Klassifikationssysteme ICD-10 (International Classification of Diseases) und DSM IV (Diagnostic and Statistical Manual) ein, der zugrunde liegende Störungsbegriff ist wesentlich weiter gefasst und deskriptiv, also nicht notwendigerweise an eine bestimmte Ätiologie gebunden. Damit werden z. B. Substanzmittelmissbrauch und -abhängigkeit in den Rang einer psychischen Störung erhoben, die über Besonderheiten des Konsums definiert wird und psychische Phänomene lediglich als dessen Folgen mitberücksichtigt. Und die sog. Störungen des Sozialverhaltens sind durch ein sich wieder- holendes und anhaltendes Muster dissozialen, aggressiven und aufsässigen Verhaltens charakterisiert. In ihre Definition gehen neben normverletzenden Verhaltensweisen (Lügen) und Delinquenz (Schulschwänzen, Weglaufen von zuhause) zusätzlich Straftatbestände (Feuerlegen, erhebliche Destruktivität gegenüber Eigentum) ein. Auch hier ist eine psychische Symptomatik nicht obligat. Diese in Kauf genommene Inkonsistenz ist eine Folge der »forensischen « Nachfrage nach Exkulpatation bzw. Dekulpatation und nach Behandlung bzw. Sicherung.

Auch die forensische Psychologie orientiert sich ganz wesentlich an den genannten Klassifikationssystemen psychischer Störungen. Dabei ist sie vornehmlich in der Grundlagenforschung tätig, die Erfassung der Persönlichkeit von Straftätern erfolgt theoriegeleitet und empirisch orientiert weitgehend über psychometrische Testverfahren sowie strukturierte (klinische) Interviews. In den aktuellen multikausalen Erklärungsmodellen von Gewalt und Aggression werden psychopathologische Phänomene nicht direkt erfasst (Köhnken, Bliesener 2002), sondern über inhaltlich recht unterschiedliche Risikofaktoren (z.B. schwieriges Temperament, Bindungsdefizite, soziale Disorganisation; vgl. Bliesener 2003). Das verwundert insofern auch nicht, als dass sich das Fachgebiet über eine empirische Herangehensweise dem Phänomen der Kriminalität nähert und versucht, Variablen zu finden, welche einen möglichst großen Varianzanteil von delinquenten Verhalten erklären können. Ausgehend von der Beschäftigung mit normalpsychologischen Prozessen markieren somit störungsrelevante Aspekte einen speziellen und eher nachrangigen Bereich. Durch diesen Forschungsansatz soll der Komplexität von Delinquenz (z.B. Entstehung und Aufrechterhaltung) aber auch der Dynamik (z.B. Veränderung oder Entwicklung) Rechnung getragen werden.

Neben Tatumständen und Charakteristika der Strafverfolgung bis in den juristischen Bereich analysiert die herkömmliche täterorientierte Kriminologie ebenfalls Tätermerkmale.

Die Kriminalsoziologie hingegen steht - ähnlich wie die Soziologie - mit ihren bevorzugten Interessenschwerpunkten: Rollenzuweisungen, 
Definitionsverzerrungen, Normentstehung und Selektionsprozesse -einer solchen Personalisierung oder gar Psychologisierung in Verbindung mit strafbaren Handlungen traditionell eher skeptisch gegenüber.

Die Bereiche Fallanalyse und Täterprofil (Hoffmann, Musolff 2000) bieten aktuell ein Kooperationsfeld für Kriminologen bzw. Kriminalisten und Vertreter der sog. Psychowissenschaften.

Innerhalb der Öffentlichen Meinung stellt sich die Verbindung von Straftaten und psychischer Störung ambivalent dar. Einerseits wird gerade bei spektakulären und besonders bei monströsen Taten der voreilige Schluss auf eine zu Grunde liegende psychische Störung gezogen („,wer so etwas tut, muss psychisch krank sein"), andererseits bestehen Argwohn und Skepsis, dass kriminelles Verhalten allzu leicht durch eine damit in Verbindung gesehene psychische Problematik bagatellisiert oder gar entschuldigt wird. Es dürfte somit weniger um den Aufklärungswert solcher Ansätze gehen, sondern deren Beurteilung im Gesamt einer moralischen Empörung.

\section{Aktueller Forschungsstand}

Obwohl es seit längerem bereits als bekannt gilt, dass psychische Störungen bei Straftätern überdurchschnittlich häufig vorkommen, liegen erst seit kurzem methodisch hinreichend abgesicherte Studien zu dieser Fragestellung vor, ja das Thema scheint im letzten Jahrzehnt besondere Beachtung gefunden zu haben.

Fazal und Danesh (2002) haben in einer Metaanalyse (62 Untersuchungen an Inhaftierten aus 12 Ländern) festgestellt, dass etwa 15\% der Probanden an sog. kernpsychiatrischen Erkrankungen wie Schizophrenie und schwerer Depression litten, Persönlichkeitsstörungen fanden sich bei $65 \%$. Erhebliche Prävalenzraten - teilweise doppelt so hoch wie bei psychiatrischen Patienten zeigten sich hinsichtlich Substanzmissbrauch und -abhängigkeit. Eine sog. Psychopathie/ psychopathy (Unfähigkeit zur Anpassung an soziale Normen mit entsprechenden Einstellungen und Verhaltensweisen, s.u.) wiesen $20 \%$ der Untersuchten auf (Andersen et al. 2000). Auch jugendliche und heranwachsende Straftäter zeigen in hohem Ausmaß v.a. Persönlichkeitsstörungen (mind. 30\%) sowie solche durch Drogenkonsum (mind. 25\%) (Köhler, Hinrichs 2004).

Wie lassen sich diese inzwischen weitgehend abgesicherten Untersuchungsergebnisse nun sinnvoll interpretieren?

Über die Weite des zu Grunde liegenden Krankheitsbegriffes wurde bereits gesprochen. Heute kann sich jeder im Internet kundig machen, ob bei seinen Kindern ein Aufmerksamkeitsdefizit-Syndrom/ADS (was ist eigentlich an einem Aufmerksamkeitsdefizit schon syndromal ?) vorliegt oder er selber - anhand eines Kriterienkataloges eingeschätzt - am Asperger-Syndrom (Autismus-Form) leidet. Wer Drogen konsumiert, muss nicht zwangsläufig psychisch krank sein, dennoch wird dies bei dem größten Teil der Konsumenten angenommen. Dafür spricht auch nicht automatisch die hohe Komorbidität mit Persönlichkeitsstörungen und depressiven Erkrankungen, die entweder vorher bereits vorhanden gewesen sein können oder auch in Zusammenhang mit einem bestimmten Lebensstil zu sehen sind.

Die genannten Klassifikationssysteme weisen i.d.R. keine Abstufung nach Schweregrad einer Störung auf, aus der sich ein Grenzwert ergeben würde, der das Vorliegen markiert oder ausschließt. Bei dem überwiegenden Anteil psychischer Störungen - insbesondere bei den Persönlichkeitsstörungen (vgl. Fiedler, 1997) - muss ohnehin von einer dimensionalen Verteilung (d.h., das jeweilige Merkmal verteilt sich in der Population annähernd kontinuierlich in seiner Ausprägung) ausgegangen werden, die eine normative Entscheidung erfordert, ab wann das entsprechende Kriterium als erfüllt gilt. Für viele Störungsbereiche liegen allerdings nur syndromale Merkmalskataloge mit »willkürlich « festgelegten »Cut-Off-Werten« zur Unterscheidung von »gestört« vs. »normal « vor, ohne dass dafür ein Vergleich mit der allgemeinen Bevölkerung die Grundlage bildet. An dieser Stelle kann weiter angemerkt werden, dass viele der einzelnen Störungskategorien der Klassifikationssysteme auf Basis von Konsensentscheidungen in Fachgremien festgelegt wurden und nicht auf der Grundlage von empirischen Untersuchungen oder Befunden. Besonders deutlich wird dies beispielsweise für den Bereich der Persönlichkeitsstörungen. Hier existieren zwischen den Systemen (ICD-10 und DSM-IV) teilweise deutliche Unterschiede (vgl. Fiedler, 1997; Köhler, 1999).

Unter methodischen Gesichtspunkten muss bedacht werden, dass die zitierte Forschung fast ausschließlich an Inhaftierten vorgenommen wurde, damit handelt es sich um eine extrem belastete Gruppe, deren Kennwerte sich keineswegs auf Straffällige insgesamt übertragen lassen. Und mit der Inhaftierung ist ein besonderer Untersuchungskontext mit speziellen (auch) psychischen Auswirkungen verbunden. Unter der Belastung durch die begangene Straftat, ggf. auch die korrespondierende Lebenssituation sowie die $\mathrm{zu}$ erwartenden Rechtsfolgen entsteht oft ein Leidensdruck, der gerade bei subjektiven Schilderungen und Selbstbeschreibungen nicht unberücksichtigt bleiben darf, jedoch schwer zu erfassen bzw. zu kontrollieren ist.

Bei vielen der angewandten Untersuchungsverfahren handelt es sich um sog. Such- oder Screening-Instrumente mit hoher Sensitivität (möglichst viele Störungen werden erfasst) bei geringer
Spezifität (Trennschärfe bzgl. unterschiedlicher Störungsformen). Dadurch dürfte der »wahre« Anteil psychischer Störungen überschätzt werden, denn um möglichst viele Ausprägungen zu erfassen, sind die Einschlusskriterien weit.

Kaum eine Untersuchung zur Häufigkeit von psychischen Störungen bei Inhaftierten relativiert diese in Bezug auf die Bevölkerungsgruppe, der diese Probanden vornehmlich entstammen. Sozial deutlich belastete Populationen weisen ohnehin schon 15-20\% psychisch Gestörter auf. Und einem aktuellen Artikel des Deutschen Ärzteblattes konnte man gar Folgendes entnehmen: "Nach dem aktuellen Gesundheitssurvey des Bundesgesundheitsministeriums bestehen bei 31 Prozent der Bevölkerung psychische Störungen. Etwa die Hälfte der Bevölkerung erlebt einmal im Leben eine psychische Krise." (Richter-Kuhlmann 2004). Angesichts solcher Befunde relativieren sich die genannten Prävalenzen für Straftäter erheblich.

Die meisten psychischen Störungen, die nach ICD-10 oder DSM-IV diagnostiziert wurden, bleiben unterhalb der Schwelle des juristisch definierten ersten (krankhafte seelische Störung) und vierten (schwere andere seelische Abartigkeit) Eingangskriteriums zur Bestimmung der Schuldfähigkeit. Hier ist die Orientierung an einem (medizinischen) Krankheitsmodell bestehen geblieben.

\section{Dissoziale Persönlichkeitsstörung und Psychopathie}

Für die im letzten Abschnitt angedeuteten Schwierigkeiten sollen kurz zwei ausgewählte Diagnosen bzw. Störungskonzepte diskutiert werden. Die dissoziale Persönlichkeitsstörung ist durch eine Missachtung sozialer Verpflichtungen, einen Mangel an Gefühlen für andere, ausgeprägte Gewalt oder herzloses Unbeteiligtsein gekennzeichnet. Damit werden moralische Defizite, bestimmte Einstellungen und Verhaltensweisen als konstituierende Merkmale genannt, zusätzlich eine geringe Veränderungswahrscheinlichkeit auch unter Repression. Eine einheitliche, dem zu Grunde liegende Theorie ist nicht $\mathrm{zu}$ erkennen, sieht man einmal von der psychiatrischen Nomenklatur der Persönlichkeitsstörungen im Sinne einer Gruppierung ab. Somit fällt es ausgesprochen schwer, dissoziale Verhaltensweisen, die eng an einen risikobehafteten Lebensstil und eine spezielle Bezugsgruppe unter Berücksichtigung besonderer biographischer Parameter gebunden sind, zu trennen von vergleichbaren Formen auf der Grundlage bestimmter relativ überdauernder Persönlichkeitsfaktoren (sog. »traits«).

Mit dem Konzept der Psychopathie von Hare (1991) ist ein Ansatz gegeben, diese Differenzierung auch konzeptuell zu gestalten. Darunter versteht man eine psychiatrische Störungsen- 
tität, die ähnliche Parameter wie die dissoziale Persönlichkeitsstörung aufweist, bei der aber weitere und extremer ausgeprägte Störungsanteile der Persönlichkeit, v.a. narzisstische eine wesentliche Rolle spielen (Huchzermeier et al. 2003). Das meist benutzte Verfahren zur Erfassung dieses Konstruktes, die PsychopathieCheckliste/PCL (Hare 1991) muss als inhaltlich und methodisch unzureichend (theoretische Verankerung zweifelhaft mit stark wertendem Charakter, tautologische Merkmalsbestimmungen aus unterschiedlichen Bereichen, fragwürdige Quantifizierung und Gewichtung) angesehen werden (Hinrichs 2003). Deshalb wäre es unangemessen und möglicherweise gefährlich, die Reichweite solcher Diagnosen zu überschätzen, da mit ihnen auch eine Festschreibung einhergeht. Andererseits bieten sie die Möglichkeit einer weiterreichenden Analyse kriminovalenter Faktoren mit den entsprechenden Möglichkeiten in Prognose und Therapie.

\section{Diskussion}

Man kann davon ausgehen, dass Straftäter überdurchschnittlich oft an psychischen Störungen leiden, vornehmlich solche der Persönlichkeit und des Drogenkonsums. Anderseits ist das Vorliegen von psychischer Störung (bei bestimmten Formen!) ein Risikofaktor für gewalttätiges Verhalten, so dass dieser Einflussfaktor unbedingt mit berücksichtigt werden muss. Weiterhin weicht das Verteilungsmuster bestimmter psychischer Störungen bei Straftätern sowohl von dem einer Normalpopulation wie auch dem psychiatrisch-psychotherapeutischer Stichproben deutlich ab. Es finden sich vorrangig sog. externalisierende Störungen, bei denen der Konfliktfokus nicht intrapsychisch, sondern interaktiv bearbeitet wird. Außerdem erhöhen soziale Widrigkeiten die Wahrscheinlichkeit für das Entstehen psychischer Probleme - auch ohne dass ein kriminelles Verhalten vorliegt.

Wie bereits dargestellt, ist die reine Prävalenzbestimmung psychischer Störungen bei Straftätern bedingt sinnvoll und unterliegt einer gewissen "Pathophilie«. Eine Eingrenzung auf behandlungsbedürftige Störungen könnte mehr Sinn machen als die Bestimmung ihrer »Krankheitswertigkeit«. Auch die bekannten Faktoren wie Leidensdruck, Therapiemotivation und die Beeinträchtigung sozialer Beziehungen sowie der Arbeitsfähigkeit geben wertvolle zusätzliche Information. Und damit wäre man wieder bei dem eingangs von Rasch (1986) erwähnten strukturell-sozialen Krankheitsbegriff angelangt. Wenn in gezielten Ansätzen jeweils das straffällige Verhalten und die psychische Problematik behandelt werden (Müller-Isberner 2002), steht das einer ganzheitlichen, personenzentrierten Wirkung nicht grundsätzlich im Wege. Es sollte aber auch deutlich gemacht werden, wo Be- handlung keine Grundlage hat und ggf. sogar zu einer Fixierung der Störung führt.

\section{Literatur:}

Aichhorn, A. (1926): Verwahrloste Jugend. Bern: Huber.

Freud, S. (1916): Einige Charaktertypen aus der psychoanalytischen Arbeit. Gesammelte Werke Bd. 10.London: Imago:364-391.

Andersen, H., Sestoft, D., Lillebaek, T., Gabrielsen, G., Hemmingsen, R., Kramp, P. (2000): A longitudinal study of prisoners on remand: psychiatric prevalence, incidence and psychopathology in solitary vs. non-solitary confinement. Acta Psychiatrica Scandinavia, 102: 19-25.

Bliesener, T. (2003): Jugenddelinquenz. Risikofaktoren, Prävention, Intervention und Prognose. Praxis der Rechtspsychologie, 2: 174-191.

Fazal, S., Danesh, J. (2002): Serious mental disorder in 23000 prisoners: a systematic review of 62 surveys. The Lancet, 359: 545-548.

Fiedler, P. (1997): Persönlichkeitsstörungen. Weinheim: PVU.

Hare, R.D. (1991): The Hare Psychopathy Checklist-Revised. Toronto: Multi-Health Systems.

Hinrichs, G. (2003): Prognosebegutachtung: Neue Ansätze oder doch "alter Wein in neuen Schläuchen«? Neue Kriminalpolitik, 15: 89-91.

Hoffmann, J., Musolff, C. (2000): Fallanalyse und Täterprofil. BKA Forschungsreihe, Band 52. Bönen: DruckVerlag Kettler.

Huchzermeier, C., Goth, N., Köhler, D., Hinrichs, G. und Aldenhoff, J. (2003): Psychopathie und Persönlichkeitsstörungen. Monatsschrift für Kriminologie und Strafrechtsreform, 86: 206215.

Köhler, D. (1999). Klassifikation und Diagnostik von Persönlichkeitsstörungen und ihre Beziehungen zum Fünf-Faktoren Modell der Persönlichkeit. Unveröffentlichte Diplomarbeit an der Universität Bielefeld. Fakultät für Psychologie und Sportwissenschaften. www.forensischepsychologie.de.

Köhler, D., Hinrichs G. (2004): Zur Prävalenz pschischer Störungen bei jugendlichen und heranwachsenden Gefangenen im Regelvollzug. In: Osterheider, M. (Hrsg.): 18. Eickelborner Fachtagung. Krank und/oder kriminell? Behandlungsund Organisationsmodelle in der Forensik. Dortmund: PschoGen Verlag, S. 174-182.

Köhnken, G. und Bliesener T. (2002): Psychologische Theorien zur Erklärung von Gewalt und Aggression. In: Ostendorf, H., Köhnken, G. und Schütze G.(Hrsg.): Agression und Gewalt. Frankfurt/M.: Peter Lang, S. 71-94.

Rasch, W. (1986): Forensische Psychiatrie. Stuttgart: Kohlhammer.

Richter-Kuhlmann, E. (2004): Psychische Störungen. Psychiater: Ohne Hausärzte geht es nicht. Deutsches Ärzteblatt, 101: 12.

Priv.Doz. Dr. Günter Hinrichs ist stellvertretender Direktor der Klinik für Kinder- und Jugendpsychiatrie und Psychotherapie, Niemannsweg 147, 24105 Kiel
TERMINAL

\section{"Gewalt in der Schule. Ausprägung, Erklärung, Prävention«}

\author{
Kriminologische Studienwoche \\ vom 7. - 11. März 2005 \\ in der Universität Hamburg
}

Das Thema »Gewalt an der Schule« wird bereits seit 20 Jahren national und international in unterschiedlicher Intensität diskutiert. Dabei fanden bislang vor allem spektakuläre Fälle (in den letzten Jahren etwa Columbine, Erfurt, Hildesheim) eine massenmediale Beachtung, die ihresgleichen sucht. Als Folge einer solchen Berichterstattung steigt phasenweise die Verunsicherung der Bevölkerung und somit die Handlungsanforderung an die Politik.

In solchen Phasen werden hitzige Debatten geführt, die jedoch in der Regel wenig über den tatsächlichen Umfang und die Bedingungen von Jugendgewalt aussagen. Langfristige Entwicklungen bleiben in der Betrachtung spektakulärer Einzelfälle ebenso im Dunkeln, wie die umfassende Analyse von Entstehungsbedingungen und die Ableitung effektiver Präventionsbemühungen.

Dennoch existieren mittlerweile zahlreiche wissenschaftliche Erkenntnisse, die wertvolle Hinweise zur Analyse der Ursachen und Eindämmung der Problematik erlauben.

In der vom Institut für Kriminologische Sozialforschung der Universität Hamburg geplanten Studienwoche sollen kriminologische und interdisziplinäre Forschungsergebnisse dargestellt und durch einen breit angelegten Dialog zwischen Wissenschaft und Praxis nutzbar gemacht werden. Hierbei führt der Bogen von den leichten und schweren Formen der Schulgewalt über die Rolle der Massenmedien bis zur Entwicklung von Strategien zur Konfliktdeeskalation und Gewaltprävention.

\section{Bewerbungsschluss:}

7. Januar 2005, Teilnahmegebühr: 252,-€

Weitere Informationen erhalten Sie von:

Ursula Kisse, Universität Hamburg, Institut für Kriminologische Sozialforschung, Allende-Platz 1, 20146 Hamburg,

Tel.: 040-428.38.6504;

Sekretariat: 04-428-38-3329

E-Mail: ursula.kisse@uni-hamburg.de oder unter:

www.rrz.uni-hamburg.de/kriminol/welcome.htm 